\title{
Association of endothelial dysfunction with sarcopenia and muscle function in a relatively young cohort of kidney transplant recipients
}

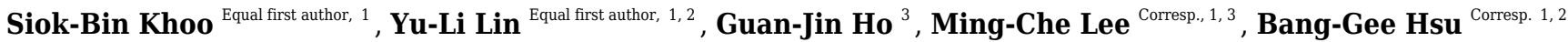 \\ ${ }^{1}$ School of Medicine, Tzu Chi University, Hualien, Taiwan \\ 2 Division of Nephrology, Hualien Tzu Chi Hospital, Buddhist Tzu Chi Medical Foundation, Hualien, Taiwan \\ 3 Department of Surgery, Hualien Tzu Chi Hospital, Buddhist Tzu Chi Medical Foundation, Hualien, Taiwan \\ Corresponding Authors: Ming-Che Lee, Bang-Gee Hsu \\ Email address: mclee1229@mail.tcu.edu.tw, gee.lily@msa.hinet.net
}

Background. Sarcopenia and endothelial dysfunction are both common among kidney transplant (KT) recipients. We aimed to evaluate the association between endothelial dysfunction and sarcopenia, as well as its individual components.

Methods. Vascular reactivity index (VRI), skeletal muscle index (SMI=skeletal muscle mass/height ${ }^{2}$ ), handgrip strength (HGS), and 6-meter usual gait speed (GS) were measured in $95 \mathrm{KT}$ recipients. Low SMI was defined as SMI less than $10 \%$ of the sex-specific reference values from Chinese adults; low HGS as HGS $<28 \mathrm{~kg}$ for men and < $18 \mathrm{~kg}$ for women; slow GS as GS below $1.0 \mathrm{~m} / \mathrm{s}$. Sarcopenia was diagnosed based on the presence of low SMI as an essential criterion, accompanied by either low HGS or slow GS. Vascular reactivity was classified as being indicative of poor ( $\mathrm{VRI}<1.0)$, intermediate $(1.0 \leq \mathrm{VRI}<2.0$ ), or good ( VRI $\geq 2.0$ ) vascular reactivity.

Results. Of the 95 patients, aged $45.2 \pm 10.9$ years, $11.6 \%$ had sarcopenia and $13.7 \%$ had poor vascular reactivity. Patients with sarcopenia were lower in body mass index $(p=0.001)$ and VRI $(p=0.041)$, and have a higher proportion of low muscle mass $(p<0.001)$, low HGS $(p<0.001)$, and slow GS $(p=0.001)$. Patients with poor vascular reactivity have a higher proportion of sarcopenia $(p=0.005)$, low HGS ( $p$ $=0.006)$, and slow GS $(p=0.029)$. Multivariate logistic regression analysis showed that patients in the poor VRI group were significantly associated with sarcopenia (odds ratio, OR $=6.17 ; 95 \%$ confidence interval, $1.06-36.04 ; p=0.043)$, comparing to those with good VRI. We further analysed the effects of VRI on individual components of sarcopenia and found that VRI predicted slow GS significantly $(O R=0.41$; $95 \% \mathrm{Cl}=0.21-0.79 ; \mathrm{p}=0.007)$, but not low SMI (OR $=1.15 ; 95 \% \mathrm{Cl}=0.53-2.49 ; \mathrm{p}=0.718)$ and HGS (OR $=$ $0.59 ; 95 \% \mathrm{Cl}=0.31-1.16 ; p=0.125)$.

Conclusions. We concluded that endothelial dysfunction is a key determinant of sarcopenia in KT recipients. Furthermore, endothelial dysfunction is more closely related to gait speed than muscle mass and strength. 


\section{Association of endothelial dysfunction with}

$9 \quad{ }^{1}$ School of Medicine, Tzu Chi University, Hualien, Taiwan

${ }^{2}$ Division of Nephrology, Hualien Tzu Chi Hospital, Buddhist Tzu Chi Medical Foundation, 11 Hualien, Taiwan

$12{ }^{3}$ Department of Surgery, Hualien Tzu Chi Hospital, Buddhist Tzu Chi Medical Foundation, 13 Hualien, Taiwan

These authors contributed equally to this work.

*Corresponding Author:

17 Ming-Che Lee and Bang-Gee Hsu

No. 707, Section 3, Chung Yang Road, 97004 Hualien, Taiwan.

Email address: mclee1229@mail.tcu.edu.tw (Ming-Che Lee); gee.lily@msa.hinet.net (Bang-Gee Hsu) 


\section{Abstract}

24 Background. Sarcopenia and endothelial dysfunction are both common among kidney transplant

25 (KT) recipients. We aimed to evaluate the association between endothelial dysfunction and 26 sarcopenia, as well as its individual components.

27 Methods. Vascular reactivity index (VRI), skeletal muscle index (SMI=skeletal muscle 28 mass/height ${ }^{2}$ ), handgrip strength (HGS), and 6-meter usual gait speed (GS) were measured in 95 29 KT recipients. Low SMI was defined as SMI less than $10 \%$ of the sex-specific reference values 30 from Chinese adults; low HGS as HGS $<28 \mathrm{~kg}$ for men and $<18 \mathrm{~kg}$ for women; slow GS as GS 31 below $1.0 \mathrm{~m} / \mathrm{s}$. Sarcopenia was diagnosed based on the presence of low SMI as an essential 32 criterion, accompanied by either low HGS or slow GS. Vascular reactivity was classified as 33 being indicative of poor $(\mathrm{VRI}<1.0)$, intermediate $(1.0 \leq \mathrm{VRI}<2.0)$, or good $(\mathrm{VRI} \geq 2.0)$ 34 vascular reactivity.

35 Results. Of the 95 patients, aged $45.2 \pm 10.9$ years, $11.6 \%$ had sarcopenia and $13.7 \%$ had poor 36 vascular reactivity. Patients with sarcopenia were lower in body mass index $(p=0.001)$ and VRI ( $p=0.041)$, and have a higher proportion of low muscle mass ( $p<0.001)$, low HGS $(p<0.001)$, and slow GS $(p=0.001)$. Patients with poor vascular reactivity have a higher proportion of sarcopenia ( $p=0.005)$, low HGS ( $p=0.006)$, and slow GS $(p=0.029)$. Multivariate logistic

40

41

42

43 regression analysis showed that patients in the poor VRI group were significantly associated with sarcopenia (odds ratio, $\mathrm{OR}=6.17 ; 95 \%$ confidence interval, $1.06-36.04 ; p=0.043$ ), comparing to those with good VRI. We further analysed the effects of VRI on individual components of sarcopenia and found that VRI predicted slow GS significantly ( $\mathrm{OR}=0.41 ; 95 \% \mathrm{CI}=0.21-0.79$; $\mathrm{p}=0.007)$, but not low SMI $(\mathrm{OR}=1.15 ; 95 \% \mathrm{CI}=0.53-2.49 ; \mathrm{p}=0.718)$ and $\mathrm{HGS}(\mathrm{OR}=0.59 ; 95 \%$ $\mathrm{CI}=0.31-1.16 ; \mathrm{p}=0.125)$.

Conclusions. We concluded that endothelial dysfunction is a key determinant of sarcopenia in KT recipients. Furthermore, endothelial dysfunction is more closely related to gait speed than muscle mass and strength.

Keywords: endothelial function, sarcopenia, muscle mass, muscle strength, gait speed, kidney transplantation. 


\section{Introduction}

55

56

57

58

59

60

61

62

63

64

65

66

67

68

69

70

71

72

73

74

75

76

77

78

79

80

81

Sarcopenia, characterized by a progressive decline of skeletal muscle mass, strength, and physical performance, is highly prevalent in patients undergoing dialysis, which leads to poor clinical outcomes (Domanski \& Ciechanowski, 2012; Fahal, 2014; Moorthi \& Avin, 2017). While kidney transplantation (KT) serves as the optimal treatment for end-stage renal disease, which improves the quality of life and lowers the mortality rate, the prevalence of sarcopenia remains higher than the general population ( Limirio et al., 2019; Kosoku et al., 2020). Chronic inflammation, reduced energy and protein intake, nutrient loss, oxidative stress, insulin resistance, and pre-existing comorbidities are well-established risk factors associated with sarcopenia (Fahal, 2014; Stenvinkel et al., 2016). Beyond these factors, vascular dysfunction is postulated to play a crucial role in the pathogenesis of sarcopenia in the rationale of high vascularization of skeletal muscle (Larsson et al., 2019).

As a key regulator of vascular homeostasis, endothelial function may be implicated in the skeletal muscle health. In chronic heart failure patients, impaired endothelial function was more frequently observed in those with sarcopenia (Dos Santos et al., 2017). In community-dwelling older women, there was a significant relationship between endothelial function and skeletal muscle strength (Yoo et al., 2018). While abnormality in endothelial function is highly prevalent in KT recipients (Yildirim et al., 2015), research over the past decade has mainly focused its role on kidney graft survival and cardiovascular outcomes ( Shoskes \& Halloran, 1996; Abedini et al., 2010; Frenay et al., 2015); there are no previous studies addressing the association of endothelial dysfunction and sarcopenia in KT recipients.

As a hallmark of endothelial dysfunction, impaired endothelial-dependent dilatation could be assessed by digital thermal monitoring (DTM), a reliable noninvasive method to measure vascular reactivity index (VRI). Poor VRI is well-documented to be correlated with atherosclerosis and predict cardiovascular risks (Naghavi et al., 2016). Thus, the objective of the present study was to explore, in KT recipients, the association of VRI with sarcopenia and its individual components. 


\section{Materials \& Methods}

83 Participants

84 This cross-sectional study was conducted in the renal transplant outpatient clinic of the 85 medical center in Hualien, Taiwan. Prevalent KT recipients receiving transplantation for more

86 than six months were recruited between September 2015 and March 2016. This study was

87 approved by the Protection of Human Subjects Institutional Review Board of Tzu-Chi University 88 and Hospital (IRB104-84-B). All enrolled patients provided written informed consent and the local ethical committee before study entry. Exclusion criteria were any KT recipients with acute 90 infection within three months, acute transplant rejection status, decompensated heart failure, and malignancy at the time of enrollment, or if they refused to provide informed consent for the 92 study. of medical records. Comorbid diseases included diabetes mellitus (DM), hypertension, hyperlipidemia, and cardiovascular (CV) disease, which comprised coronary artery disease, myocardial infarction, arrhythmias, or congestive heart failure. The immunosuppressive drug use history included the use of tacrolimus, mycophenolate mofetil (MMF), steroid, rapamycin, and cyclosporine.

\section{Blood pressure and endothelial function measurements}

Blood pressure was measured using standard mercury sphygmomanometers after 10minute rest. Endothelial function was assessed by DTM (Endothelix Inc., Houston, TX, USA), which is highly reproducible, fully automated, and non-operator dependent. The measurements were performed after an overnight fast of at least 10 hours. After placing the blood pressure cuffs over both bare upper arms and fixing the skin temperature sensors to both of the patient's index fingers, DTM of both hands was performed during 3 minutes of stabilization, 2 minutes of cuff inflation to $50 \mathrm{mmHg}$ greater than systolic blood pressure, and 5 minutes of deflation. When the cuff was released, blood flow rushed into the forearm and hand, causing a temperature rebound in the fingertip, which was directly proportional to the reactive hyperemia response. During the reactive hyperemia period, the VENDYS software calculated VRI, which assessed the maximum difference between the observed temperature rebound curve and the zero-reactivity curve (

111 Schier et al., 2013; Naghavi et al., 2016). The coefficient of repeatability of temperature rebound

112 and area under the curve previously reported were $2.4 \%$ and $2.8 \%$ (Ahmadi et al., 2011). 
113 Vascular reactivity was classified as being indicative of poor (VRI $<1.0)$, intermediate $(1.0 \leq$

114 VRI $<2.0$ ), or good (VRI $\geq 2.0$ ) vascular reactivity (Naghavi et al. 2016).

115 Skeletal muscle mass, handgrip strength, and usual gait speed measurements

116 Bodyweight was measured with the patients wearing light indoor clothing and body height 117 with the patient standing barefoot or stockings. BMI was calculated as weight $/ \mathrm{height}^{2}\left(\mathrm{~kg} / \mathrm{m}^{2}\right)$. A

118 single-frequency bioimpedance device (Tanita BC 706DB, Tanita Corporation, Tokyo, Japan)

119 was used for the measurement of skeletal muscle mass. Skeletal muscle index (SMI) was

120 calculated as skeletal muscle mass $/ h_{e} \operatorname{ghht}^{2}\left(\mathrm{~kg} / \mathrm{m}^{2}\right)$. Body fat mass was measured using the same

121 device. Low SMI was defined as SMI $<16.5 \mathrm{~kg} / \mathrm{m}^{2}$ in men and $<14.2 \mathrm{~kg} / \mathrm{m}^{2}$ in women, according

122 to the SMI less than $10 \%$ of the sex-specific reference values from Chinese adults (Jin et al., 123 2019).

124 Handgrip strength (HGS) was assessed using a Jamar Plus Digital Hand Dynamometer (SI 125 Instruments Pty Ltd, Hilton, Australia), with a precision of 1 kilogram $(\mathrm{kg})$. All patients were 126 instructed to hold the dynamometer in both hands and to squeeze it as hard as they could, with 127 the elbow flexed at 90 degrees, in an upright standing position to keep their arms at their sides.

128 The measurement was repeated three times in both arms with a rest of one minute before the next 129 measure, and the maximum value was adopted for further analysis. Low handgrip strength was 130 classified as HGS $<28 \mathrm{~kg}$ for men and $<18 \mathrm{~kg}$ for women, according to the criteria of Asia 131 Working Group for Sarcopenia (Chen et al., 2020). Usual gait speed (GS) was measured by 132 walking for 6 meters at a comfortable pace. Slow GS was defined as GS below $1.0 \mathrm{~m} / \mathrm{s}$ in all 133 patients (Chen et al., 2020). The same trained operator conducted all procedures.

134

135

136

137

138

139

140

141

142

143

\section{Definition of sarcopenia}

Diagnoses of sarcopenia in our participants were based on the presence of low muscle mass as an essential criterion, accompanied by either low HGS or slow GS ( Cruz-Jentoft et al., 2019; Chen et al., 2020).

\section{Biochemical investigations}

From each patient, we collected approximately $5 \mathrm{ml}$ of fasting blood samples. About $1 \mathrm{ml}$ for hemoglobin ( $\mathrm{Hb}$ ) (Sysmex K-1000, Sysmex American, Mundelein, IL, USA) and $4 \mathrm{ml}$ for the others were promptly centrifuged at 3,000 g for 10 minutes. Serum levels of creatinine, fasting glucose, total cholesterol (TCH), triglycerides, and phosphate were determined by using an autoanalyzer (Siemens Advia 1800, Siemens Healthcare GmbH, Henkestr, Germany). The estimated 
144 GFR was calculated based on the CKD-EPI Creatinine equation (Inker et al., 2012). Serum 145 levels of intact parathyroid hormone (PTH) were measured by an autoanalyzer (Diagnostic 146 Systems Laboratories, Webster, Texas, USA).

\section{Statistical analysis}

148 Assuming a 10\% prevalence of sarcopenia in our KT recipients and a mean VRI difference 149 of 0.5 between sarcopenia and non-sarcopenia (standard deviation 0.5 in both groups), with an 150 alpha level of 0.05 , a total of at least 90 patients should be enrolled to achieve a power of $80 \%$. 151 The continuous data distribution was examined by using the Kolmogorov-Smirnov test.

152 Variables with normal distribution were expressed as mean \pm standard deviation and analyzed by 153 the Student's independent t-test or Analysis of Variance (ANOVA) test, whereas those not 154 normally distributed were expressed as medians (interquartile ranges) and analyzed by the 155 Mann-Whitney U test or Kruskal-Wallis test. Categorical variables were expressed as number $156(\%)$ and analyzed by Chi-square test. Multiple comparisons between VRI categories were made 157 by Bonferroni test. Univariate and multivariate logistic regression was used to determine if VRI 158 is associated with sarcopenia and its individual components. In addition to age and gender, the 159 variables with significant differences between sarcopenia and non-sarcopenia groups were 160 regarded as potential confounders and were adjusted in the multivariate models. A $p$-value of 161 less than 0.05 (two-tailed) was considered statistically significant. The statistical analysis was 162 performed with SPSS 19.0 software (SPSS, Chicago, IL, USA).

163

164

165

166

167

168

169

170

171

172

173

174

\section{5}

\section{Results}


A total of 95 patients were enrolled in the study, which consisted of 46 males (48.4\%) and

177

178

179

180

181

182

183

184

185

186

187

188

189

190

191

192

193

194

195

196

197

198

199

200

201

202

203

204

205

49 females $(51.6 \%)$. The mean age of the patients was $45.2 \pm 10.9$ years, and the median time from transplantation was 72.0 months (interquartile range 27.0-111.0). The prevalences of DM, HTN, hyperlipidemia, and CV disease were $45.3 \%, 38.9 \%, 41.1 \%$, and $17.9 \%$, respectively. Among them, 48 (50.5\%), 34 (35.8\%), and 13 (13.7\%) of them were classified as having good, intermediate, and poor vascular reactivity, respectively.

The distribution of low SMI, low HGS, slow GS, and sarcopenia overall and by different VRI categories are depicted in Figure 1. Overall, the prevalence of low SMI, low HGS, and slow GS was $33.7 \%, 22.1 \%$, and $36.8 \%$ in our KT recipients. Sarcopenia was identified in $11.6 \%$ of all patients. Notably, among the three VRI groups, the prevalence of low SMI, low HGS, slow GS, and sarcopenia were all higher in patients with VRI $<1.0$.

The baseline characteristics of all patients, with and without sarcopenia, are showed in Table 1. Patients with sarcopenia were lower in BMI $(p=0.001)$ and VRI $(p=0.041)$, and have a higher proportion of low muscle mass $(p<0.001)$, low HGS $(p<0.001)$, and slow GS $(p=0.001)$. There were no differences between patients with and without sarcopenia in terms of age, gender, time from transplantation, smoking status, blood pressure, laboratory data, the presence of comorbidities, and the use of medications.

The clinical characteristics of the $95 \mathrm{KT}$ recipients with good, intermediate, or poor vascular reactivity are presented in Table 2 . Patients with poor vascular reactivity have a higher proportion of sarcopenia $(p=0.005)$, low HGS $(p=0.006)$, and slow GS $(p=0.029)$. There was no significant difference in age, gender, time from transplantation, smoking status, blood pressure, laboratory data among the three groups. There were also no differences in the distribution of comorbidities or the use of medications.

Table 3 demonstrated the univariate and multivariate logistic regression of sarcopenia among KT recipients. In addition to BMI, patients in the poor VRI group were significantly associated with sarcopenia (odds ratio, $\mathrm{OR}=6.88 ; 95 \%$ confidence interval, $\mathrm{CI}=1.51-31.29$; $p=0.013$ ), comparing to those with good VRI. This association remained unchanged after adjustment $(\mathrm{OR}=6.17 ; 95 \% \mathrm{CI}=1.06-36.04 ; p=0.043)$. However, while treating VRI as a continuous variable, the association between VRI and sarcopenia didn't achieve significance after adjustment $(\mathrm{OR}=0.46 ; 95 \% \mathrm{CI}=0.20-1.04 ; p=0.062)$. 
206 Table 4 depicted the associations of VRI with low SMI, HGS, and slow GS individually. 207 After full adjustment, VRI entered as a continuous variable independently predicted slow GS 208 significantly $(\mathrm{OR}=0.41 ; 95 \% \mathrm{CI}=0.21-0.79 ; p=0.007)$, but not low HGS (OR $=0.59 ; 95 \%$ $209 \mathrm{CI}=0.31-1.16 ; p=0.125)$ and $\mathrm{SMI}(\mathrm{OR}=1.15 ; 95 \% \mathrm{CI}=0.53-2.49 ; p=0.718)$. Similar results 210 were achieved when VRI was entered as a categorical variable. 


\section{Discussion}

212 The primary findings of the present study are that lower BMI and poor VRI were two 213 independent factors associated with sarcopenia in our KT recipients. Furthermore, among the three 214 components of sarcopenia, VRI appeared to predict GS, but no SMI and HGS.

215 Few previous studies investigated the link between endothelial dysfunction and sarcopenia 216 and its individual components. In chronic heart failure patients, those with sarcopenia had a 217 lower baseline and peak reactive hyperemia blood flow in the forearm and leg than those without 218 sarcopenia. This reactive hyperemia was closely associated with physical performance, included 219 peak VO2 and 6-minute walk distance (Dos Santos et al., 2017). In community-dwelling older women, endothelial dysfunction independently predicted handgrip strength weakness (Yoo et al., 2018). Elevated serum levels of asymmetric dimethylarginine, a surrogate biomarker of

222

223

224

225

226

227

228

229

230

231

232

233

234

235

236

237

238

239

240

241 endothelial dysfunction, were not only associated with lower muscle strengths and slower GS among elderly individuals (Obayashi et al., 2016) but also with reduced physical performance in the 10-repetition sit-to-stand test in prevalent hemodialysis patients (Pajek et al., 2018). In accordance with aforementioned findings, our study, for the first time, showed a significant association between endothelial dysfunction and sarcopenia in KT recipients.

Skeletal muscle mass, HGS, and GS, the three components of sarcopenia, may have different clinical relevance. Among our KT recipients, we interestingly found that VRI was more closely related to GS than HGS and SMI. This finding indicated that endothelial dysfunction may have more adverse impacts on physical performance than on muscle mass and strength. Physiologically, the performance of gait speed test is more dependent upon enhanced local blood flow, compared to the production of maximal handgrip strength. Consistent with our finding, in elderly and patients with pre-dialysis chronic kidney disease, endothelial dysfunction is associated with frailty, a clinical syndrome characterized by a decline in physiological reserve and poor physical performance (Alonso-Bouzón et al., 2014; Mansur et al., 2015).

Several possible mechanisms explained the crosslink between endothelial dysfunction and sarcopenia in KT recipients. First, skeletal muscle microcirculation plays a vital role in skeletal muscle health (Hendrickse \& Degens, 2019). Endothelial dysfunction was shown to impair microcirculation of skeletal muscle and downregulate vascular endothelial growth factor expression, which compromises angiogenesis and hampers muscle regeneration (Latroche et al., 2015). In vitro study, treatment of asymmetric dimethylarginine, an endothelial inhibitor of nitric 
242 oxide synthesis, results in increased protein degradation in cultured C2C12 myotubes (Zhou et 243 al., 2009). Second, impaired endothelial function in skeletal muscle may reduce the delivery of 244 dietary amino acids to skeletal muscle fibers and impair skeletal muscle protein synthesis (Moro 245 et al., 2016). Third, emerging evidence showed myokines, which are hundreds of cytokines and 246 proteins secreted by skeletal muscle in response to muscle contraction, exert autocrine, paracrine, 247 and endocrine effects on various systems, including the vascular system (Severinsen \& Pedersen, 248 2020). For example, follistatin-related protein 1, a muscle-secreted glycoprotein, has been shown 249 to regulate endothelial cell function and blood vessel growth in skeletal muscle through a nitric250 oxide synthase-dependent mechanism (Ouchi et al., 2008). Fourth, endothelial dysfunction 251 compromises cardiovascular health, which may manifest as muscle weakness and poor physical

252 253

254 255 256 257 258 259 260 261 262 263 264 265 266

267 268 269

270 271 272 performance. Finally, endothelial dysfunction and sarcopenia shared some common pathogenic mechanisms, such as inflammation, insulin resistance, and oxidative stress, which could explain the close link to each other (Fahal, 2014; Goligorsky, 2015).

Apart from endothelial dysfunction, BMI was another relevant factor strongly associated with sarcopenia in our KT recipients. Consistent with our findings, Kosoku et al. showed that BMI is a reliable nutritional marker for predicting sarcopenia in KT recipients, which has a good discrimination performance (Kosoku et al., 2020).

Surprisingly, aging is recognized as an irrefutable predictor for sarcopenia in the geriatric population (Yamada et al., 2013; Han et al., 2016), but not in our study. This discrepancy may be explained by the relatively young age of our enrolled participants.

This is the first study to report the association between endothelial dysfunction and sarcopenia in KT recipients. However, several significant limitations need to be considered. First, the total sample size was relatively small, and a small proportion was categorized as having sarcopenia and poor VRI, which could affect the precision of estimation. Second, we directly measured vascular reactivity but didn't measure some biomarkers of endothelium dysfunction, such as asymmetric dimethylarginine and Soluble Vascular Cell Adhesion Molecule-1. Third, a bioimpedance device was used for the measurement of skeletal muscle mass. Computed tomography and magnetic resonance imaging, the gold standard for the assessment of skeletal muscle mass, were not used. Fourth, inflammatory markers, such as C-reactive protein and interleukin-6, were not available in this study. Fifth, the causal relationships between endothelial function and sarcopenia couldn't be established by the cross-sectional design. Finally, the 
273 participants in this study were relatively young, and our results may not be generalizable to 274 elderly KT recipients.

\section{Conclusions}

276 Our study showed a significant association between endothelial dysfunction and 277 sarcopenia in KT recipients, which implicated that endothelial dysfunction may be involved in 278 the pathogenesis of sarcopenia. Furthermore, endothelial dysfunction is more closely related to 279 physical performance than muscle mass and strength. Further longitudinal studies investigating 280 the changes among endothelial function, skeletal muscle mass, and function are warranted to 281 establish the causal relationship. 


\section{References}

284 Abedini S, Meinitzer A, Holme I, Marz W, Weihrauch G, Fellstrom B, Jardine A, and Holdaas

285

286

287

288

289

290

291

292

293

294

295

296

297

298

299

300

301

302

303

304

305

306

307

308

309

310

311

312

313

H. 2010. Asymmetrical dimethylarginine is associated with renal and cardiovascular outcomes and all-cause mortality in renal transplant recipients. Kidney Int 77:44-50. 10.1038/ki.2009.382

Ahmadi N, McQuilkin GL, Akhtar MW, Hajsadeghi F, Kleis SJ, Hecht H, Naghavi M, Budoff M. 2011. Reproducibility and variability of digital thermal monitoring of vascular reactivity. Clin Physiol Funct Imaging 31(6):422-8.

Alonso-Bouzón C, Carcaillon L, García-García FJ, Amor-Andrés MS, El Assar M, and Rodríguez-Mañas L. 2014. Association between endothelial dysfunction and frailty: the Toledo Study for Healthy Aging. Age (Dordr) 36:495-505. 10.1007/s11357-013-9576-1

Chen L-K, Liu L-K, Woo J, Assantachai P, Auyeung T-W, Bahyah KS, Chou M-Y, Chen L-Y, Hsu P-S, and Krairit O. 2014. Sarcopenia in Asia: consensus report of the Asian Working Group for Sarcopenia. Journal of the American Medical Directors Association 15:95101.

Chen LK, Woo J, Assantachai P, Auyeung TW, Chou MY, Iijima K, Jang HC, Kang L, Kim M, Kim S, Kojima T, Kuzuya M, Lee JSW, Lee SY, Lee WJ, Lee Y, Liang CK, Lim JY, Lim WS, Peng LN, Sugimoto K, Tanaka T, Won CW, Yamada M, Zhang T, Akishita M, and Arai H. 2020. Asian Working Group for Sarcopenia: 2019 Consensus Update on Sarcopenia Diagnosis and Treatment. J Am Med Dir Assoc 21:300-307 e302. 10.1016/j.jamda.2019.12.012

Cruz-Jentoft AJ, Baeyens JP, Bauer JM, Boirie Y, Cederholm T, Landi F, Martin FC, Michel JP, Rolland Y, Schneider SM, Topinkova E, Vandewoude M, Zamboni M, and European Working Group on Sarcopenia in Older P. 2010. Sarcopenia: European consensus on definition and diagnosis: Report of the European Working Group on Sarcopenia in Older People. Age Ageing 39:412-423. 10.1093/ageing/afq034

Cruz-Jentoft AJ, Bahat G, Bauer J, Boirie Y, Bruyere O, Cederholm T, Cooper C, Landi F, Rolland Y, Sayer AA, Schneider SM, Sieber CC, Topinkova E, Vandewoude M, Visser M, Zamboni M, Writing Group for the European Working Group on Sarcopenia in Older P, and the Extended Group for E. 2019. Sarcopenia: revised European consensus on definition and diagnosis. Age Ageing 48:16-31. 10.1093/ageing/afy 169 
314 Domanski M, and Ciechanowski K. 2012. Sarcopenia: a major challenge in elderly patients with

315

316

317

318

319

320

321

322

323

324

325

326

327

328

329

330

331

332

333

334

335

336

337

338

339

340

341

342

343

344 end-stage renal disease. J Aging Res 2012:754739. 10.1155/2012/754739

Dos Santos MR, Saitoh M, Ebner N, Valentova M, Konishi M, Ishida J, Emami A, Springer J, Sandek A, Doehner W, Anker SD, and von Haehling S. 2017. Sarcopenia and Endothelial Function in Patients With Chronic Heart Failure: Results From the Studies Investigating Comorbidities Aggravating Heart Failure (SICA-HF). J Am Med Dir Assoc 18:240-245. 10.1016/j.jamda.2016.09.006

Fahal IH. 2014. Uraemic sarcopenia: aetiology and implications. Nephrol Dial Transplant 29:1655-1665. 10.1093/ndt/gft070

Frenay A-RS, van den Berg E, de Borst MH, Beckmann B, Tsikas D, Feelisch M, Navis G, Bakker SJL, and van Goor H. 2015. Plasma ADMA associates with all-cause mortality in renal transplant recipients. Amino Acids 47:1941-1949. 10.1007/s00726-015-2023-0

Goligorsky MS. 2015. Pathogenesis of endothelial cell dysfunction in chronic kidney disease: a retrospective and what the future may hold. Kidney Research and Clinical Practice 34:76-82. https://doi.org/10.1016/j.krcp.2015.05.003

Han P, Kang L, Guo Q, Wang J, Zhang W, Shen S, Wang X, Dong R, Ma Y, Shi Y, Shi Z, Li H, Li C, Ma Y, Wang L, and Niu K. 2016. Prevalence and Factors Associated With Sarcopenia in Suburb-dwelling Older Chinese Using the Asian Working Group for Sarcopenia Definition. The Journals of Gerontology Series A: Biological Sciences and Medical Sciences 71:529-535. 10.1093/gerona/glv108

Hendrickse P, and Degens H. 2019. The role of the microcirculation in muscle function and plasticity. J Muscle Res Cell Motil 40:127-140. 10.1007/s10974-019-09520-2

Inker LA, Schmid CH, Tighiouart H, Eckfeldt JH, Feldman HI, Greene T, Kusek JW, Manzi J, Van Lente F, Zhang YL, Coresh J, Levey AS; CKD-EPI Investigators. 2012. Estimating glomerular filtration rate from serum creatinine and cystatin C. N Engl J Med 367:20-9. 10.1056/NEJMoa1114248

Jin M, Du H, Zhang Y, Zhu H, Xu K, Yuan X, Pan H, and Shan G. 2019. Characteristics and reference values of fat mass index and fat free mass index by bioelectrical impedance analysis in an adult population. Clinical Nutrition 38:2325-2332. https://doi.org/10.1016/j.clnu.2018.10.010

Kosoku A, Uchida J, Nishide S, Kabei K, Shimada H, Iwai T, Maeda K, Hanayama Y, Ishihara 
345

346

347

348

349

350

351

352

353

354

355

356

357

358

359

360

361

362

363

364

365

366

367

368

369

370

371

372

373

374

375

T, Naganuma T, Takemoto Y, and Nakatani T. 2020. Association of sarcopenia with phase angle and body mass index in kidney transplant recipients. Scientific Reports 10:266. 10.1038/s41598-019-57195-z

Larsson L, Degens H, Li M, Salviati L, Lee YI, Thompson W, Kirkland JL, Sandri M. 2019. Sarcopenia: Aging-Related Loss of Muscle Mass and Function. Physiol Rev. 99:427-511. 10.1152/physrev.00061.2017

Latroche C, Gitiaux C, Chrétien F, Desguerre I, Mounier R, and Chazaud B. 2015. Skeletal Muscle Microvasculature: A Highly Dynamic Lifeline. Physiology 30:417-427. 10.1152/physiol.00026.2015

Limirio LS, Santos HO, dos Reis AS, and de Oliveira EP. 2019. (Dis) Agreement between the first and the recent European consensus on definition and diagnosis for sarcopenia in kidney transplant patients. European Journal of Clinical Nutrition. 10.1038/s41430-0190535-5

Mansur HN, Lovisi JCM, Colugnati FAB, Raposo NRB, Fernandes NMdS, and Bastos MG. 2015. Association of frailty with endothelial dysfunction and its possible impact on negative outcomes in Brazilian predialysis patients with chronic kidney disease. $B M C$ nephrology 16:157. 10.1186/s12882-015-0150-1

Moorthi RN, and Avin KG. 2017. Clinical relevance of sarcopenia in chronic kidney disease. Curr Opin Nephrol Hypertens 26:219-228. 10.1097/MNH.0000000000000318

Moro T, Ebert SM, Adams CM, and Rasmussen BB. 2016. Amino Acid Sensing in Skeletal Muscle. Trends in endocrinology and metabolism: TEM 27:796-806. 10.1016/j.tem.2016.06.010

Naghavi M, Yen AA, Lin AW, Tanaka H, and Kleis S. 2016. New Indices of Endothelial Function Measured by Digital Thermal Monitoring of Vascular Reactivity: Data from 6084 Patients Registry. Int J Vasc Med 2016:1348028. 10.1155/2016/1348028

Obayashi K, Saeki K, Maegawa T, Sakai T, Kitagawa M, Otaki N, Kataoka H, and Kurumatani N. 2016. Association of Serum Asymmetric Dimethylarginine With Muscle Strength and Gait Speed: A Cross-Sectional Study of the HEIJO-KYO Cohort. Journal of Bone and Mineral Research 31:1107-1113. 10.1002/jbmr.2773

Ouchi N, Oshima Y, Ohashi K, Higuchi A, Ikegami C, Izumiya Y, and Walsh K. 2008. Follistatin-like 1, a secreted muscle protein, promotes endothelial cell function and 
376

377

378

379

380

381

382

383

384

385

386

387

388

389

390

391

392

393

394

395

396

397

398

399

400

401

402

403

404

405

406

revascularization in ischemic tissue through a nitric-oxide synthase-dependent mechanism. J Biol Chem 283:32802-32811. 10.1074/jbc.M803440200

Pajek M, Jerman A, Osredkar J, Ponikvar JB, and Pajek J. 2018. Association of Uremic Toxins and Inflammatory Markers with Physical Performance in Dialysis Patients. Toxins $10: 403$.

Schier R, Marcus HE, Mansur E, Lei X, El-Zein R, Mehran R, Purugganan R, Heir JS, Riedel B, and Gottumukkala V. 2013. Evaluation of digital thermal monitoring as a tool to assess perioperative vascular reactivity. $J$ Atheroscler Thromb 20:277-286.

Severinsen MCK, and Pedersen BK. 2020. Muscle-Organ Crosstalk: The Emerging Roles of Myokines. Endocr Rev 41:594-609. 10.1210/endrev/bnaa016

Shoskes DA, and Halloran PF. 1996. Delayed Graft Function in Renal Transplantation: Etiology, Management and Long-term Significance. The Journal of Urology 155:1831-1840. https://doi.org/10.1016/S0022-5347(01)66023-3

Stenvinkel P, Carrero JJ, von Walden F, Ikizler TA, and Nader GA. 2016. Muscle wasting in end-stage renal disease promulgates premature death: established, emerging and potential novel treatment strategies. Nephrol Dial Transplant 31:1070-1077. 10.1093/ndt/gfv122

Yamada M, Nishiguchi S, Fukutani N, Tanigawa T, Yukutake T, Kayama H, Aoyama T, and Arai H. 2013. Prevalence of Sarcopenia in Community-Dwelling Japanese Older Adults. Journal of the American Medical Directors Association 14:911-915. 10.1016/j.jamda.2013.08.015

Yildirim T, Yilmaz R, Altindal M, Turkmen E, Arici M, Altun B, and Erdem Y. 2015. Endothelial dysfunction in renal transplant recipients: role of vitamin D and fibroblast growth factor-23. Transplantation proceedings 47:343-347.

10.1016/j.transproceed.2014.12.023

Yoo JI, Kim MJ, Na JB, Chun YH, Park YJ, Park Y, Hah YS, Ha YC, and Park KS. 2018. Relationship between endothelial function and skeletal muscle strength in community dwelling elderly women. J Cachexia Sarcopenia Muscle 9:1034-1041. $10.1002 / j \operatorname{csm} .12340$

Zhou QG, Zhou M, Hou FF, and Peng X. 2009. Asymmetrical dimethylarginine triggers lipolysis and inflammatory response via induction of endoplasmic reticulum stress in cultured adipocytes. American Journal of Physiology-Endocrinology And Metabolism 296:E869- 
E878. 10.1152/ajpendo.91011.2008

408 


\section{Table 1 (on next page)}

Demographic and clinical characteristics of 95 kidney transplantation recipients, with and without sarcopenia. 
1 Table 1. Demographic and clinical characteristics of 95 kidney transplantation recipients, with 2 and without sarcopenia.

\begin{tabular}{|c|c|c|c|c|}
\hline Characteristics & $\begin{array}{c}\text { All } \\
(n=95)\end{array}$ & $\begin{array}{l}\text { Sarcopenia } \\
\qquad(\mathrm{n}=11)\end{array}$ & $\begin{array}{l}\text { Non-sarcopenia } \\
\qquad(N=84)\end{array}$ & $P$ \\
\hline \multicolumn{5}{|l|}{ Demographics } \\
\hline Age (years) & $45.2 \pm 10.9$ & $44.1 \pm 11.6$ & $45.3 \pm 10.8$ & 0.724 \\
\hline \multicolumn{5}{|l|}{ Gender, $n(\%)$} \\
\hline Male & $46(48.4)$ & $6(54.5)$ & $40(47.6)$ & \multirow{2}{*}{0.666} \\
\hline Female & $49(51.6)$ & $5(45.5)$ & $44(52.4)$ & \\
\hline $\begin{array}{l}\text { Time from transplantation } \\
\text { (months) }\end{array}$ & $72.0(84.0)$ & $61.0(120.0)$ & $72.0(75.0)$ & 0.789 \\
\hline Current smoking, $\mathrm{n}(\%)$ & $7(7.4)$ & $0(0)$ & $7(8.3)$ & 0.320 \\
\hline \multicolumn{5}{|l|}{ Examination } \\
\hline SBP (mmHg) & $144.0 \pm 18.7$ & $138.4 \pm 17.9$ & $144.7 \pm 18.8$ & 0.292 \\
\hline DBP (mmHg) & $84.1 \pm 12.5$ & $80.4 \pm 10.3$ & $84.6 \pm 12.7$ & 0.296 \\
\hline VRI & $1.9 \pm 0.8$ & $1.4 \pm 0.8$ & $2.0 \pm 0.8$ & $0.041^{*}$ \\
\hline BMI $\left(\mathrm{kg} / \mathrm{m}^{2}\right)$ & $23.3(5.8)$ & $20.6(3.5)$ & $24.1(5.5)$ & $0.001 *$ \\
\hline Body fat (\%) & $29.1 \pm 9.2$ & $25.2 \pm 8.6$ & $29.6 \pm 9.2$ & 0.133 \\
\hline Low SMI, $n(\%)$ & $32(33.7)$ & $11(100.0)$ & $21(25.0)$ & $<0.001 *$ \\
\hline Low HGS, $n(\%)$ & $21(22.1)$ & $7(63.6)$ & $14(16.7)$ & $<0.001 *$ \\
\hline Slow GS, $n(\%)$ & $35(36.8)$ & $9(81.8)$ & $26(31.0)$ & $0.001 *$ \\
\hline \multicolumn{5}{|l|}{ Laboratory data } \\
\hline $\mathrm{Hb}(\mathrm{g} / \mathrm{L})$ & $120(36)$ & $120(40)$ & $120(40)$ & 0.487 \\
\hline $\mathrm{TCH}(\mathrm{mmol} / \mathrm{L})$ & $4.77(1.32)$ & $4.74(1.50)$ & $4.77(1.24)$ & 0.629 \\
\hline Triglyceride (mmol/L) & $1.39(1.05)$ & $1.06(1.16)$ & $1.41(1.06)$ & 0.250 \\
\hline Glucose (mmol/L) & $5.33(1.33)$ & $5.22(2.39)$ & $5.33(1.29)$ & 0.439 \\
\hline eGFR $\left(\mathrm{mL} / \mathrm{min} / 1.73 \mathrm{~m}^{2}\right)$ & $59.0 \pm 26.6$ & $63.9 \pm 32.4$ & $58.3 \pm 25.9$ & 0.513 \\
\hline Phosphate (mmol/L) & $1.07 \pm 0.26$ & $1.07 \pm 0.26$ & $1.07 \pm 0.26$ & 0.913 \\
\hline Intact PTH (pmol/L) & $10.24(10.86)$ & $8.87(15.97)$ & $10.27(10.52)$ & 0.981 \\
\hline \multicolumn{5}{|l|}{ Diseases, $n(\%)$} \\
\hline $\mathrm{DM}$ & $43(45.3)$ & $4(36.4)$ & $39(46.4)$ & 0.528 \\
\hline HTN & $37(38.9)$ & $4(36.4)$ & $33(39.3)$ & 0.852 \\
\hline Hyperlipidemia & $39(41.1)$ & $5(45.5)$ & $34(40.5)$ & 0.752 \\
\hline $\mathrm{CV}$ disease & $17(17.9)$ & $3(27.3)$ & $14(16.7)$ & 0.388 \\
\hline
\end{tabular}




\section{Medications, $n(\%)$}

\begin{tabular}{lcccc} 
Tacrolimus & $64(67.4)$ & $8(72.7)$ & $56(66.7)$ & 0.687 \\
MMF & $61(64.2)$ & $5(45.5)$ & $56(66.7)$ & 0.168 \\
Steroid & $78(82.1)$ & $8(72.7)$ & $70(83.3)$ & 0.388 \\
Rapamycin & $9(9.5)$ & $2(18.2)$ & $7(8.3)$ & 0.294 \\
Cyclosporine & $17(17.9)$ & $1(9.1)$ & $16(19.0)$ & 0.418 \\
\hline
\end{tabular}

3

The cortinuous variables with normal distribution were expressed as mean \pm standard deviation, whereas those not normally distributed were express $\varnothing \mathrm{d}$ as medians (interquartile ranges).

SBP, syGtolic blood pressure; DBP, diastolic blood pressure; VRI, vascular reactivity index; BMI, body mass index; SMI, skeletal muscle index; HGS, handgrip strength; GS, gait speed; Hb, hemoglobin; TCH, total cholesterol; eGFR, estimated glomerular filtration rate; PTH, parathy\&oid hormone; DM, diabetes mellitus; HTN, hypertension; CV, cardiovascular; MMF, mycophenolate mofetil.

$* P<0.05$ was considered statistically significant.

10 
Table 2 (on next page)

Clinical characteristics of the study population stratified by vascular reactivity index categories 
1 Table 2. Clinical characteristics of the study population stratified by vascular reactivity index 2 categories

\begin{tabular}{|c|c|c|c|c|}
\hline \multirow[t]{2}{*}{ Characteristics } & \multicolumn{3}{|c|}{ Vascular reactivity index } & \multirow[b]{2}{*}{$\boldsymbol{P}$} \\
\hline & $\begin{array}{c}\text { Good } \\
(\mathrm{n}=48)\end{array}$ & $\begin{array}{l}\text { Intermediate } \\
\qquad(\mathrm{n}=\mathbf{3 4})\end{array}$ & $\begin{array}{c}\text { Poor } \\
(n=13)\end{array}$ & \\
\hline \multicolumn{5}{|l|}{ Demographics } \\
\hline Age (years) & $44.9 \pm 10.8$ & $44.7 \pm 11.1$ & $47.5 \pm 10.9$ & 0.721 \\
\hline \multicolumn{5}{|l|}{ Gender, $n(\%)$} \\
\hline Male & $21(43.8)$ & $17(50.0)$ & $8(61.5)$ & \multirow{2}{*}{0.509} \\
\hline Female & $27(56.3)$ & $17(50.0)$ & $5(38.5)$ & \\
\hline $\begin{array}{l}\text { Time from transplantation } \\
\text { (months) }\end{array}$ & $65.0(77.0)$ & $86.5(64.0)$ & $27.0(63.0)$ & 0.061 \\
\hline Current smoking, $n(\%)$ & $2(4.2)$ & $3(8.8)$ & $2(15.4)$ & 0.359 \\
\hline \multicolumn{5}{|l|}{ Examination } \\
\hline SBP (mmHg) & $142.7 \pm 18.6$ & $145.0 \pm 20.5$ & $146.1 \pm 14.3$ & 0.784 \\
\hline DBP $(\mathrm{mmHg})$ & $85.1 \pm 12.7$ & $82.8 \pm 13.2$ & $83.8 \pm 9.8$ & 0.723 \\
\hline VRI & $2.5 \pm 0.5$ & $1.6 \pm 0.3^{\mathrm{a}}$ & $0.4 \pm 0.3^{b, c}$ & $<0.001 *$ \\
\hline BMI $\left(\mathrm{kg} / \mathrm{m}^{2}\right)$ & $23.9(7.1)$ & $23.6(3.9)$ & $21.1(6.5)$ & 0.204 \\
\hline Body fat (\%) & $29.4 \pm 10.0$ & $30.2 \pm 7.6$ & $25.3 \pm 9.7$ & 0.265 \\
\hline Sarcopenia, $n(\%)$ & $4(8.3)$ & $2(5.9)$ & $5(38.5)^{b, c}$ & $0.005^{*}$ \\
\hline Low SMI, $n(\%)$ & $17(35.4)$ & $8(23.5)$ & $7(53.8)$ & 0.135 \\
\hline Low HGS, $n(\%)$ & $13(27.1)$ & $2(5.9)^{a}$ & $6(46.2)^{c}$ & $0.006^{*}$ \\
\hline Slow GS, $n(\%)$ & $14(29.2)$ & $12(35.3)$ & $9(69.2)^{b}$ & $0.029 *$ \\
\hline \multicolumn{5}{|l|}{ Laboratory data } \\
\hline $\mathrm{Hb}(\mathrm{g} / \mathrm{L})$ & $120(37)$ & $116(40)$ & $130(40)$ & 0.434 \\
\hline $\mathrm{TCH}(\mathrm{mmol} / \mathrm{L})$ & $4.73(1.31)$ & $4.82(1.51)$ & $4.74(1.26)$ & 0.540 \\
\hline Triglyceride $(\mathrm{mmol} / \mathrm{L})$ & $1.29(1.07)$ & $1.44(0.99)$ & $1.72(1.06)$ & 0.583 \\
\hline Glucose (mmol/L) & $5.27(1.00)$ & $5.55(2.22)$ & $5.22(1.50)$ & 0.577 \\
\hline $\mathrm{eGFR}\left(\mathrm{mL} / \mathrm{min} / 1.73 \mathrm{~m}^{2}\right)$ & $61.5 \pm 25.4$ & $55.1 \pm 29.3$ & $59.7 \pm 23.6$ & 0.570 \\
\hline Phosphate (mmol/L) & $1.07 \pm 0.26$ & $1.10 \pm 0.29$ & $1.07 \pm 0.23$ & 0.815 \\
\hline Intact PTH (pmol/L) & $10.05(9.47)$ & $10.84(11.17)$ & $10.60(15.19)$ & 0.965 \\
\hline \multicolumn{5}{|l|}{ Diseases, $n(\%)$} \\
\hline $\mathrm{DM}$ & $20(41.7)$ & $18(52.9)$ & $5(38.5)$ & 0.521 \\
\hline HTN & $17(35.4)$ & $15(44.1)$ & $5(38.5)$ & 0.728 \\
\hline Hyperlipidemia & $17(35.4)$ & $15(44.1)$ & $7(53.8)$ & 0.440 \\
\hline
\end{tabular}



CV disease
$9(18.8)$
$6(17.6)$
$2(15.4)$
0.960

Medications, $n$ (\%)

$\begin{array}{lcccc}\text { Tacrolimus } & 32(66.7) & 24(70.6) & 8(61.5) & 0.830 \\ \text { MMF } & 31(64.6) & 22(64.7) & 8(61.5) & 0.977 \\ \text { Steroid } & 40(83.3) & 28(82.4) & 10(76.9) & 0.866 \\ \text { Rapamycin } & 3(6.3) & 5(14.7) & 1(7.7) & 0.424 \\ \text { Cyclosporine } & 11(22.9) & 3(8.8) & 3(23.1) & 0.227\end{array}$

The continuous variables with normal distribution were expressed as mean \pm standard deviation, whereas those not normally distributed were expressłd as medians (interquartile ranges).

Vasculas reactivity was classified as being indicative of poor $(\mathrm{VRI}<1.0)$, intermediate $(1.0 \leq \mathrm{VRI}<2.0)$, or good $(\mathrm{VRI} \geq 2.0)$ vascular reactiviby.

${ }^{\text {a }} \mathrm{p}<0.05$, comparison between intermediate and good VRI.

${ }^{\mathrm{b}} \mathrm{p}<0 . \$ 5$, comparison between poor and good VRI.

${ }^{c} \mathrm{p}<0 . \$ 5$, comparison between poor and intermediate VRI.

SBP, syQtolic blood pressure; DBP, diastolic blood pressure; VRI, vascular reactivity index; BMI, body mass index; SMI, skeletal muscle index;HGS, handgrip strength; GS, gait speed; Hb, hemoglobin; TCH, total cholesterol; eGFR, estimated glomerular filtration rate; PTH, parathyzoid hormone; DM, diabetes mellitus; HTN, hypertension; CV, cardiovascular; MMF, mycophenolate mofetil. ${ }^{*} p<0105$ is considered as statistically significant.

14 


\section{Table 3(on next page)}

Univariate and multivariate logistic regression analysis of factors associated with sarcopenia among 95 kidney transplantation recipients 
1 Table 3. Univariate and multivariate logistic regression analysis of factors associated with sarcopenia 2 among 95 kidney transplantation recipients

\begin{tabular}{|c|c|c|c|c|c|}
\hline \multirow{2}{*}{\multicolumn{2}{|c|}{ Variables }} & \multicolumn{2}{|l|}{ Unadjusted $^{\mathrm{a}}$} & \multicolumn{2}{|l|}{ Adjusted $^{b}$} \\
\hline & & Odds ratio $(95 \% \mathrm{CI})$ & $p$ value & Odds ratio $(95 \% \mathrm{CI})$ & $p$ value \\
\hline \multicolumn{6}{|c|}{ VRI entered as a categorical variable } \\
\hline \multicolumn{2}{|c|}{ BMI $\left(\mathrm{kg} / \mathrm{m}^{2}\right)$} & $0.64(0.47-0.87)$ & $0.004 *$ & $0.62(0.43-0.91)$ & $0.014^{*}$ \\
\hline \multicolumn{6}{|c|}{ VRI groups } \\
\hline \multicolumn{2}{|c|}{ Good } & \multicolumn{2}{|l|}{1 (reference) } & \multicolumn{2}{|l|}{1 (reference) } \\
\hline \multicolumn{2}{|c|}{ Intermediate } & $0.69(0.12-3.99)$ & 0.688 & $1.22(0.17-8.57)$ & 0.845 \\
\hline \multicolumn{2}{|c|}{ Poor } & $6.88(1.51-31.29)$ & $0.013 *$ & $6.17(1.06-36.04)$ & $0.043 *$ \\
\hline \multicolumn{6}{|c|}{ VRI entered as a continuous variable } \\
\hline \multicolumn{2}{|c|}{ BMI $\left(\mathrm{kg} / \mathrm{m}^{2}\right)$} & $0.64(0.47-0.87)$ & $0.004 *$ & $0.62(0.41-0.86)$ & $0.006 *$ \\
\hline \multicolumn{2}{|c|}{ VRI } & $0.44(0.20-0.98)$ & $0.044^{*}$ & $0.46(0.20-1.04)$ & 0.062 \\
\hline \multicolumn{6}{|l|}{3} \\
\hline \multicolumn{6}{|c|}{0.085 for VRI entered as a } \\
\hline \multicolumn{6}{|c|}{5 categorical and continuous variable, respectively. } \\
\hline \multicolumn{6}{|c|}{6 b Age, gender, BMI, and VRI were adopted in the adjusted model. Nagelkerke's R-squared in the adjusted model } \\
\hline 7 & \multicolumn{5}{|c|}{ was 0.369 when VRI was entered as a categorical variable and 0.357 when treating VRI as a continuous variable. } \\
\hline 8 & BMI, body mass inder & reactivity index. & & & \\
\hline 9 & ${ }^{*} p<0.05$ is considere & y significant. & & & \\
\hline 10 & & & & & \\
\hline
\end{tabular}




\section{Table 4 (on next page)}

Multivariate logistic regression analysis of the associations of VRI with low SMI, HGS, and slow GS in 95 kidney transplantation recipients. 
1 Table 4. Multivariate logistic regression analysis of the associations of VRI with low SMI, HGS, and 2 slow GS in 95 kidney transplantation recipients.

\begin{tabular}{|c|c|c|c|c|c|c|}
\hline \multirow[b]{2}{*}{ Variables } & \multicolumn{2}{|c|}{ Low SMI } & \multicolumn{2}{|c|}{ Low HGS } & \multicolumn{2}{|c|}{ Slow GS } \\
\hline & $\begin{array}{c}\text { Odds ratio } \\
(95 \% \mathrm{CI})\end{array}$ & $p$ value & $\begin{array}{c}\text { Odds ratio } \\
(95 \% \mathrm{CI})\end{array}$ & $p$ value & $\begin{array}{l}\text { Odds ratio } \\
(95 \% \text { CI })\end{array}$ & $p$ value \\
\hline
\end{tabular}

VRI entered as a categorical variable

\section{VRI groups}

Good

Intermediate

$0.88(0.24-3.23) \quad 0.850$

$0.17(0.03-0.81) \quad 0.026^{*}$

$1.42(0.53-3.80)$

0.481

Poor

$1.87(0.25-14.12) \quad 0.544$

$2.67(0.70-10.23)$

0.152

$7.30(1.73-30.83)$

$0.007 *$

\section{VRI entered as a continuous variable}

\section{VRI}

$$
1.15(0.53-2.49)
$$

0.718

$0.59(0.31-1.16)$

0.125

$0.41(0.21-0.79)$

3

4 Age, gender, BMI, and VRI were adjusted in the models.

5 VRI, vascular reactivity index; SMI, skeletal muscle index; HGS, handgrip strength; GS, gait speed.

$6 \quad * p<0.05$ is considered as statistically significant. 
Figure 1

Distribution of low SMI, HGS, slow GS, and sarcopenia in 95 kidney transplantation recipients, all and stratified by VRI categories.

Error bars represent $95 \%$ confidence interval.

VRI, vascular reactivity index; SMI, skeletal muscle index; HGS, handgrip strength; GS, gait speed

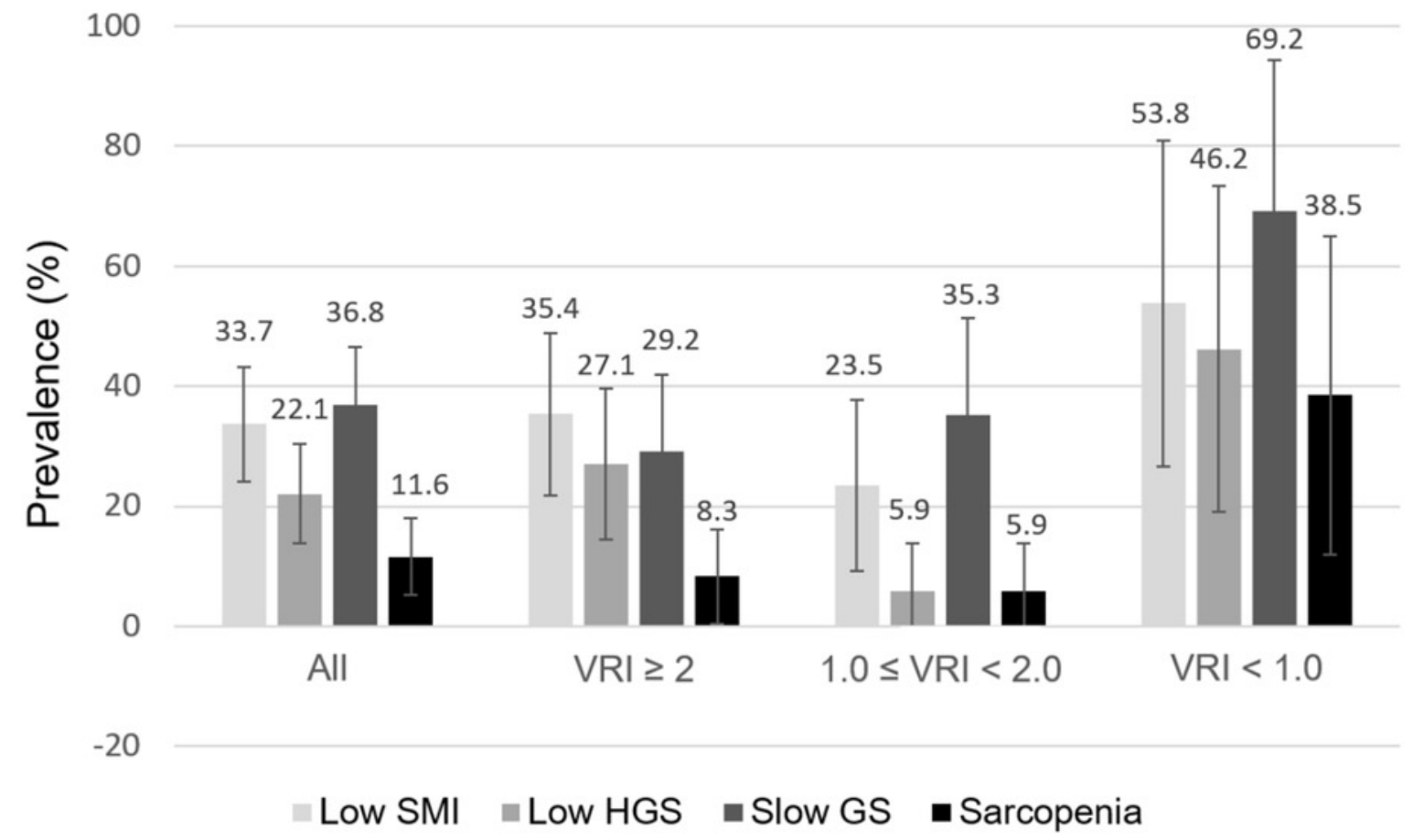

